\title{
Reply to S. Fuselier et al.'s comment on "Energetic particle sounding of the magnetospheric cusp with ISEE-1"
}

\author{
B. M. Walsh ${ }^{1}$, K. E. Whitaker ${ }^{2}$, T. A. Fritz ${ }^{1}$, J. Chen ${ }^{1}$, and M. Klida ${ }^{1}$ \\ ${ }^{1}$ Center for Space Physics, Boston University, Boston, MA, USA \\ ${ }^{2}$ Department of Astronomy, Yale University, New Haven, CT, USA
}

Received: 30 August 2008 - Revised: 30 November 2008 - Accepted: 10 December 2008 - Published: 26 January 2009

\section{Introduction}

In their comment, Fuselier et al. (2008, hereafter FPT) provide an alternative interpretation to the study done by Whitaker et al. (2007, hereafter WFC). WFC concludes that energetic ions observed in the cusp were accelerated locally, while FPT argues that the ions could have been accelerated at the bow shock and then transported to the cusp. The two analyses interpret observations made by the International Sun Earth Explorer (ISEE)-1 on 30 October 1978 as the spacecraft travelled through the high altitude cusp. ISEE-1 observed an area of depressed and turbulent magnetic field as well as increased energetic $(>10 \mathrm{keV})$ ion and electron flux.

FPT identifies the quasi-parallel bow shock as the location of particle acceleration. To show this FPT presents a model that traces particles along magnetic field lines from the cusp to the solar wind at the bow shock. This model argues energized particles from the bow shock gain entry to the magnetosphere through low latitude reconnection, propagate along reconnected field lines to the ionosphere, mirror, and return to high latitudes where they are observed flowing anti-parallel to the magnetic field by ISEE-1. We agree with the FPT model to the extent that with these IMF conditions, reconnection opens the cusp to the solar wind plasma, and newly reconnected field lines will convect tailward, but observations do not support particles being energized at the bow shock and transported to the cusp. We present three inconsistencies between the FPT model and the observations made by ISEE-1: (1) the absence of parallel flowing particles, (2)

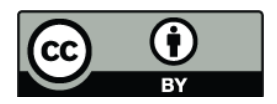

Correspondence to: B. M. Walsh (bwalsh@bu.edu) the lack of a time-energy dispersion, and (3) the presence of energetic electrons.

\section{Particle flow direction}

The first problem is that we would expect to see particles flowing parallel to the magnetic field line at some point as the satellite transitions from the magnetosphere to the magnetosheath and eventually crosses the bow shock. This is never detected. Figure 3 in FPT shows the IMF passing through the bow shock and being draped against the magnetopause. The IMF field lines then reconnect equatorward of ISEE-1, providing a connection between the solar wind and the ionosphere. FPT reports "The low and high energy ions propagate to the ionosphere along the newly open field lines, mirror, and return to high latitudes." In order for the region of enhanced particle flux to be observed for $2 \mathrm{~h}$, this must be a continuous process. If the particles are continuously flowing parallel to the magnetic field lines while they are crossing the bow shock, travelling through the magnetosheath and draping the magnetopause, we would expect to observe this as ISEE-1 travels through these regions.

The general magnetic field conditions inside and outside the magnetopause remain the same as ISEE- 1 crosses the magnetopause and travel through most of the magnetosheath (unil $B_{z}$ turns positive near 02:00 UT on 31 October, as shown in Fig. 1), so the process should not stop, yet no enhanced particle flux is observed from when ISEE-1 leaves the cusp/magnetosphere at 18:30 UT to when it crosses the bow shock at 04:15 UT on 31 October 1978. Figure 2 shows the pitch angle of the electron plasma $(10 \mathrm{eV}-2 \mathrm{keV})$ as well as the energetic ions $(24-44.5 \mathrm{keV})$ and electrons (22.5$39 \mathrm{keV}$ ) observed by ISEE-1 during this outbound pass. Energetic ions and electrons were measured with the Medium

Published by Copernicus Publications on behalf of the European Geosciences Union. 


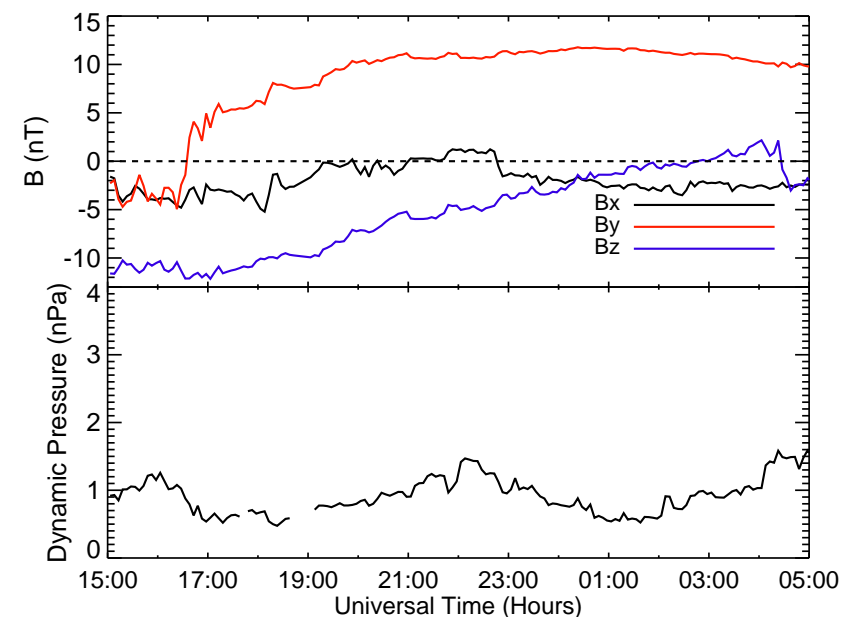

Fig. 1. Upstream solar wind parameters measured by ISEE-3 on 3031 October 1978 . The values are time-delay $(58 \mathrm{~min})$ propagated from the location of ISEE-3 to the magnetopause. Top panel provides the magnetic field components while the bottom panel is the dynamic pressure which remains low and relatively constant. The periods of interest include the time between 16:00-18:00 UT when ISEE-1 transited the high altitude cusp and then exited through the magnetopause, and a period from 18:00-04:15 UT when ISEE-1 remained in the magnetosheath until it crossed the bow shock.

Energy Particle Experiment (MEPE) (Williams et al., 1978) with $36 \mathrm{~s}$ resolution detecting over all $4 \pi$ steradian. Low energy electrons were measured with the Electron Spectrometer Experiment (Ogilvie et al., 1978) with $48 \mathrm{~s}$ resolution. While ISEE-1 is in the magnetosheath, the electron measurments show particles are close to perpendicular to the field and slightly anti-parallel. At no time was there energetic ions, electrons or lower energy electrons observed flowing parallel to the magnetic field.

Although the energetic particles make up a small part of the total plasma during this event, studying their properties is important, as observations show their behavior is often different than that of the low energy particles. Figure 2 in FTP shows the angle between the low energy electron bulk flow velocity and the local magnetic field vector. FTP highlights three points of short time duration where the angle peaks near $135^{\circ}$ and uses this to argue the high energy particles exhibit the same motion as the low energy particles. These three peaks last only several minutes each and are not representative of the low energy flow of the entire event. Again in Fig. 2 of FPT, during the extended time period between the first two peaks (16:18-17:00 UT), the angle is close to and often below $90^{\circ}$, indicating low energy plasma flowing parallel to the magnetic field vector. This is very different from what's observed through the high energy particles during the same time period. The high energy ion pitch angle distributions shown in the bottom panel of Fig. 2 in FTP show strong perpendicular and antiparallel $\left(90^{\circ}\right.$ and $\left.165^{\circ}\right)$ flux enhancments close to an order of magnitude greater than that of the parallel flux. Both of these observations are consistent with a low energy plasma population flowing parallel to magnetic field lines threading the cusp while particles energized in the cusp are observed flowing antiparallel to the magnetic field at high latitudes. During a few short some time periods, the behavior of the low energy electrons may be consistant with that of the higher energy particles, but the discrepancies make it important to focus on the high energy ion and electron measurments to find their origin.

\section{Time-energy dispersion}

The second inconsistency between the FPT model and the observations is that we see no obvious time-energy dispersion in the plasma. If ions are energized at the bow shock up to $30 R_{E}$ from the subsolar location (e.g. $X_{\mathrm{gsm}}=-30 R_{E}$ ) and travel along a flux tube to arrive in the cusp, there would be a time-energy dispersion signature. The ion flux measurements in Fig. 3 show the first four energy channels of the MEPE detector with flux separated into the pitch angles $\left(15^{\circ}\right.$, $\left.90^{\circ}, 165^{\circ}\right)$. The measurements show no visible time-energy dispersion signature.

\section{Energetic electrons}

Studies of particle energization at the quasi-parallel bow shock show Fermi acceleration of incident solar wind ions up to $\sim 150 \mathrm{keV}$ (e.g. Ipavich et al., 1981; Mobius et al., 1987; Gosling et al., 1989); however, this mechanism can not efficiently accelerate electrons (Lee, 1982). In Sect. 4, FTP argues the energetic ions observed in the cusp are energized at the bow shock and goes on to conclude that "No local acceleration is required to maintain the energetic particle population observed by ISEE-1." This conclusion refers to the energetic ion population but does not address the presense of energetic electrons.

While in the cusp diamagnetic cavity, ISEE-1 observes enhanced ion flux with energies up to $210 \mathrm{keV}$, as well as energetic electron flux showing two orders of magnitude increase for energies up to $39 \mathrm{keV}$. The presence of energetic electrons is unexplained by a bow shock source. The pitch angle of the electrons give us more information. Although the energetic electrons are centered around a pitch angle of $90^{\circ}$ throughout most of the event, during the time period between 18:17 and 18:22 UT the electron pitch angle indicates particles flowing anti-parallel to the magnetic field. This is again interrepted as particles flowing away from a local source earthward of the spacecraft. A bow shock source may be capable of producing ions at the observed energies as FPT argues, but a bow shock source is not sufficient to explain both the presence of energetic ions and electrons observations by ISEE-1. The existance of the two can not be separated in this event.

It is unlikely that the energetic electrons could have drifted to the cusp from another source. Although more energetic 


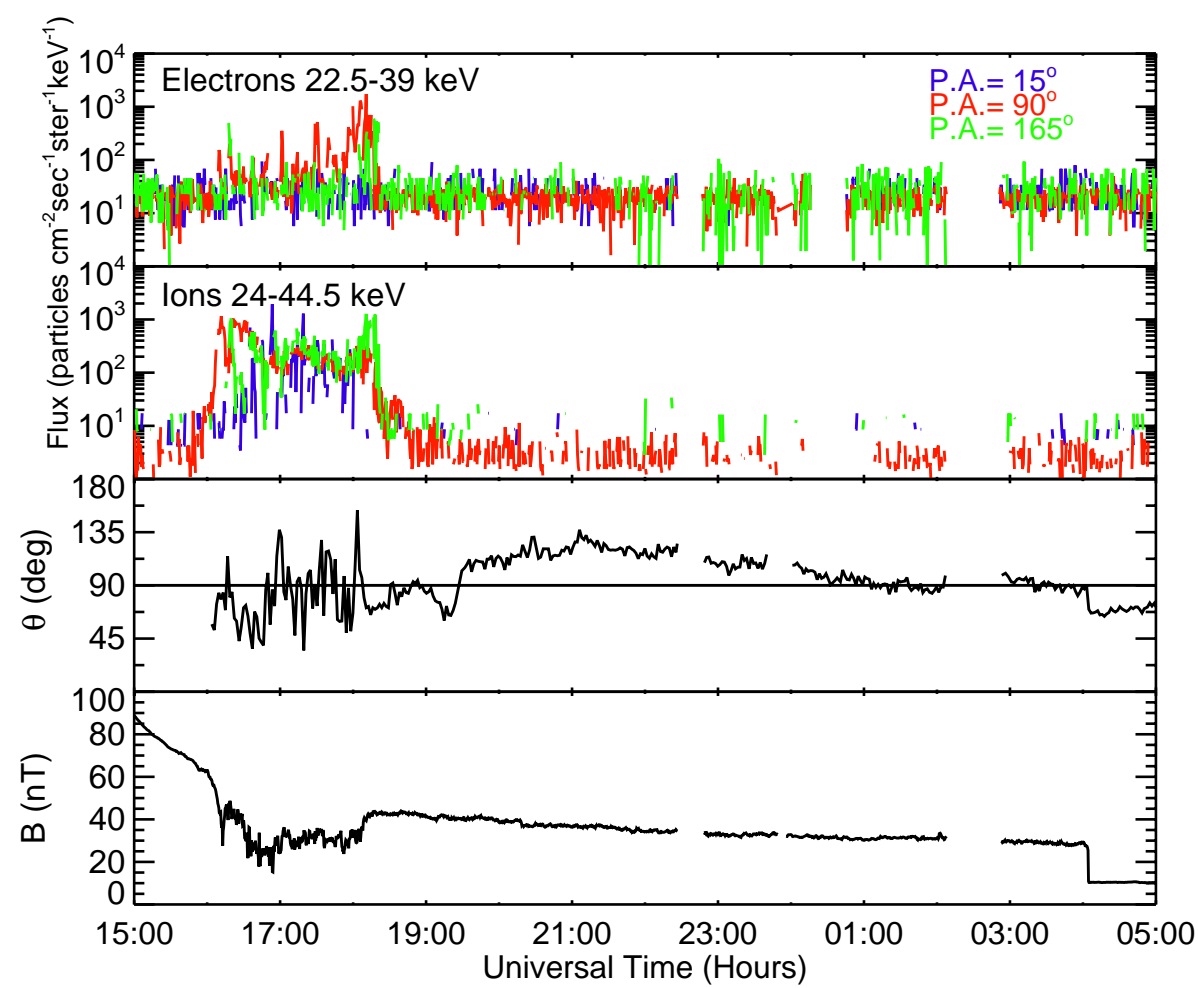

Fig. 2. Measurements made from the event on 30 October 1978 to after ISEE-1 crosses the bow shock on the following day. From top to bottom the panels are electrons (22.5-39 keV) from MEPE, then energetic ions (24-44.5 keV) from MEPE, both sorted by pitch angle. The third panel from the top is the angle between the electron $(10 \mathrm{eV}-2 \mathrm{keV})$ bulk flow direction, as measured from the Electron Spectrometer Experiment, and the magnetic field vector. The bottom panel is the magnitude of the local magnetic field provided with $4 \mathrm{~s}$ resolution.

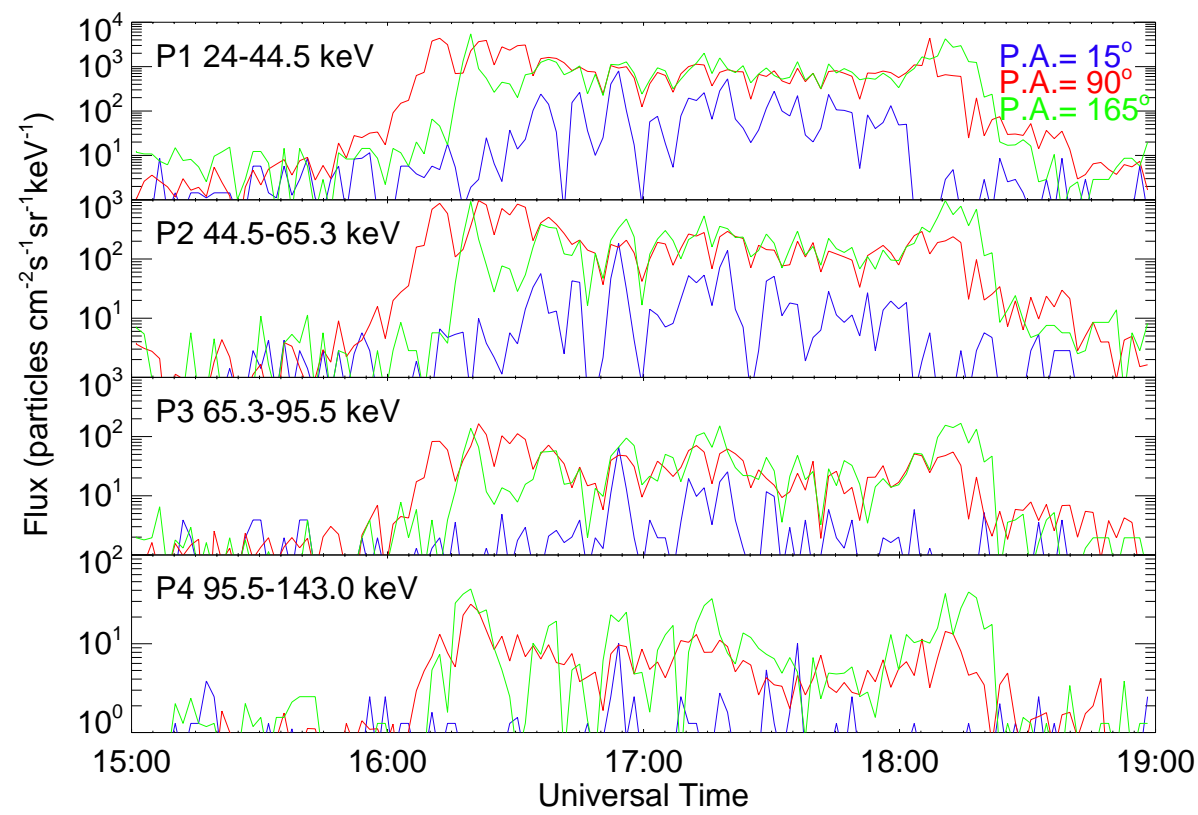

Fig. 3. Taken from Whitaker et al. (2006). From top to bottom, each panel shows ion flux with increasing energy measured with MEPE on ISEE-1 on 30 October 1978 . The particle flux is sorted by pitch angle with a tolerance of $5^{\circ}$. Note that the flux enhancements begin and finish at the same time for each energy channel. 
electron enhancments are observed during the end of the event, the electron flux enhancments are measured during the same time period we see the enhanced energetic ion flux. A similar cusp crossing by ISEE-1 was studied by Walsh et al. (2007). During the Walsh et al. (2007) event, the spacecraft observed both energetic ions and electrons as well as a depressed and turbulent magnetic field. The study showed a strong correlation between enhancments in the energetic electron and ion flux, indicating the two must be from the same source. If the particles were from two different sources and are then transported to the cusp, we would not expect such a strong correlation between when the peaks in particle flux to occur.

\section{Conclusions}

The dynamics of particle acceleration in the cusp have been investigated through several studies (e.g. Chen and Fritz, 1998; Chen, 2008; Vogiatzis et al., 2008), but more work is needed to fully understand a source that could produce both ions and electrons with the observed energies. Independent of the full dynamics of a cusp energization source, the observations of particles in this region give strong evidence that particles are accelerated locally. We exclude the bow shock as a source for the energetic particles due to its inability to explain all of the observed signatures such as (1) the absense of energetic particles and plasma flowing parallel to the magnetic field line as ISEE- 1 crosses the magnetopause and travels through the magnetosheath, (2) the lack of a time-energy dispersion from such a distance, and (3) the presense of energetic electrons. Local energization is consistent with the observations and likely to be the source of the energetic particles in the cusp.

Acknowledgements. The authors would like to acknowledge the CDAweb service for making the Electron Spectrometer Experiment from ISEE-1 available to the community. This work has stemmed out of research funded by the NASA grants NAG5 - 2575, NAG5 7677, NAG5 - 7841, NAG5 - 9562, NAG5 - 11397.

Topical Editor I. A. Daglis thanks two anonymous referees for their help in evaluating this paper.

\section{References}

Chen, J.: Evidence for particle acceleration in the magnetospheric cusp, Ann. Geophys., 26, 1993-1997, 2008, http://www.ann-geophys.net/26/1993/2008/.

Chen, J. and Fritz, T. A.: Correlation of cusp MeV helium with turbulent ULF power spectra and its implications, Geophys. Res. Lett., 25, 4113-4116, 1998.

Gosling, J. T., Thomsen, M. F., Bame, S. J., and Russell, C. T.: On the source of diffuse, suprathermal ions observed in the vicinity of the Earth's bow shock, J. Geophys. Res., 94, 3555-3563, 1989.

Ipavich, F. M., Galvin, A. B., Gloeckler, G., Scholer, M., and Hovestadt, D.: A Statistical Study of Ions Observed Upstream of the Earth's Bow Shock: Energy Spectra, Composition, and Spatial Variation, J. Geophys. Res. 86, 4337-4342, 1981.

Lee, M. A.: Coupled hydromagneticwave excitation and ion acceleration upstream of the Earth's bow shock, J. Geophys. Res., 87, 5063-5080, 1982.

Möbius, E., Scholer, M., Schopke, N., et al.: The distribution function of diffuse ions and the magnetic field power spectrum upstream of the Earth's bow shock, Geophys. Res. Lett., 14, 681684, 1987.

Ogilvie, K.W., Scudder, J. D., and Doong, H.: The electron spectrometer experiment on ISEE-1, IEEE Trans. Geosci. GE-16, 261-265, 1978.

Vogiatzis, I. I., Sarris, T. E., Sarris, E. T., Santolík, O., Dandouras, I., Robert, P., Fritz, T. A., Zong, Q.-G., and Zhang, H.: Cluster observations of particle acceleration up to supra-thermal energies in the cusp region related to low-frequency wave activity possible implications for the substorm initiation process, Ann. Geophys., 26, 653-669, 2008, http://www.ann-geophys.net/26/653/2008/.

Whitaker, K. E., Chen, J., and Fritz, T. A.: CEP populations observed by ISEE 1, Geophys. Res. Lett., 33, L23105, doi:10.1029/2006GL027731, 2006.

Whitaker, K. E., Fritz, T. A., Chen, J., and Klida, M.: Energetic particle sounding of the magnetospheric cusp with ISEE-1, Ann. Geophys., 25, 1175-1182, 2007, http://www.ann-geophys.net/25/1175/2007/.

Williams, D. J., Keppler, E., Fritz, T. A., et al.: The ISEE 1 and 2 Medium Energy Particles Experiment, IEEE Trans. Geosci. Electron., 16, 270-280, 1978.

Walsh, B. M., Fritz, T. A., Lender, N. M., Chen, J., and Whitaker, K. E.: Energetic particles observed by ISEE-1 and ISEE-2 in a cusp diamagnetic cavity on 29 September 1978, Ann. Geophys., 25, 2633-2640, 2007, http://www.ann-geophys.net/25/2633/2007/. 